\title{
Techniques available in reconstructive surgery of the eyelid
}

\author{
BYRON SMITH AND F. P. ENGLISH
}

Departments of Ophthalmology and Ophthalmic Plastic Surgery, Manhattan Eye, Ear and Throat Hospital, New Tork

Reconstructive surgery of the eyelids is undertaken in cases in which lid restoration is indicated after the excision of tumours, or after injury, or because of congenital abnormalities.

Utilization of the avulsed flap is recommended after trauma if this is at all feasible. The repair should be performed as soon as possible, but reasonable delay is no contraindication and a successful take with a time lapse of up to 36 hours has been seen at this clinic in the current year. Figs $I$ and 2 illustrate the preoperative and postoperative features of a case treated by Dr. Alston P. Callahan, in which 12 hours elapsed before the avulsed flap was replaced.

(I)

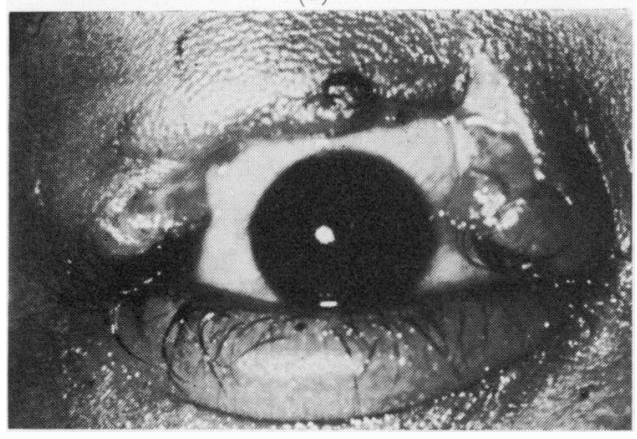

(2)

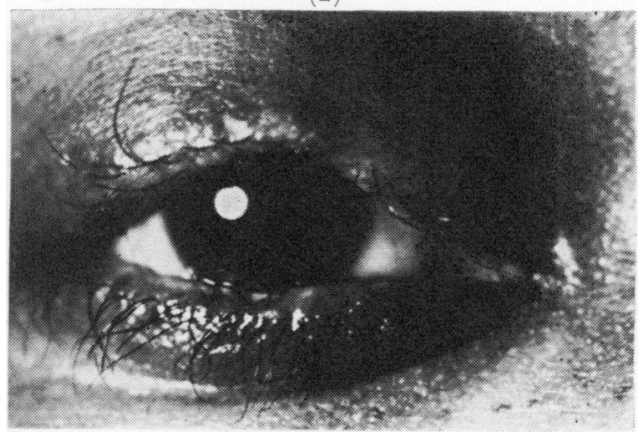

When excision of eyelid structure is indicated, the surgeon should remember that up to one-third of the horizontal dimension can be sacrificed with impunity.

Manipulation of the temporal portion with a skin hook allows the surgeon to estimate if undue tension will be present on the suture line. In such circumstances cantholysis (Figs 3, 4, and 5) may be performed by direct exposure, or by the indirect method which produces added flexibility, allowing the incision to be closed adequately. By this technique, a wedge of up to $10 \mathrm{~mm}$. in size has been resected and closed adequately.

Closure is effected by inserting buried interrupted 6-o silk sutures in the pre-tarsal fascia (Fig. 6). Three sutures of similar size are used in the intermarginal strip. The first approximates the posterior margin and the second the anterior border; the third suture controls the accurate alignment of the grey line. The needle of each suture should emerge through the lid substance about $2 \mathrm{~mm}$. from the line of incision. Before completing the tie on the second suture, the other tied border sutures are passed through this to avoid postoperative abrasion of the cornea. The accurate approximation of these three sutures guarantees a successful surgical result. Finally, the skin incision is closed with fine silk or chromic catgut. 
(3)

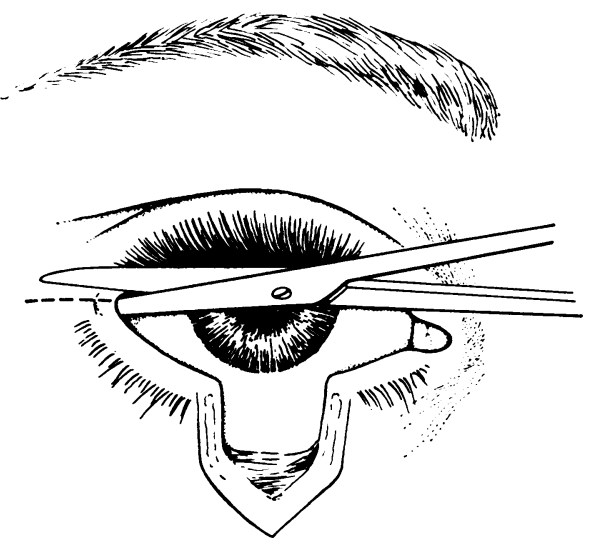

(5)

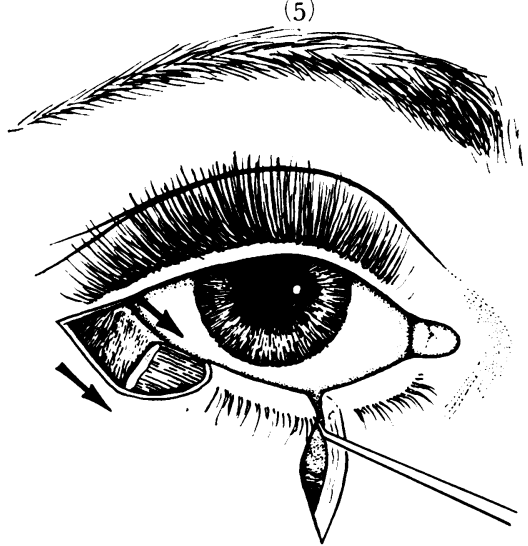

(4)

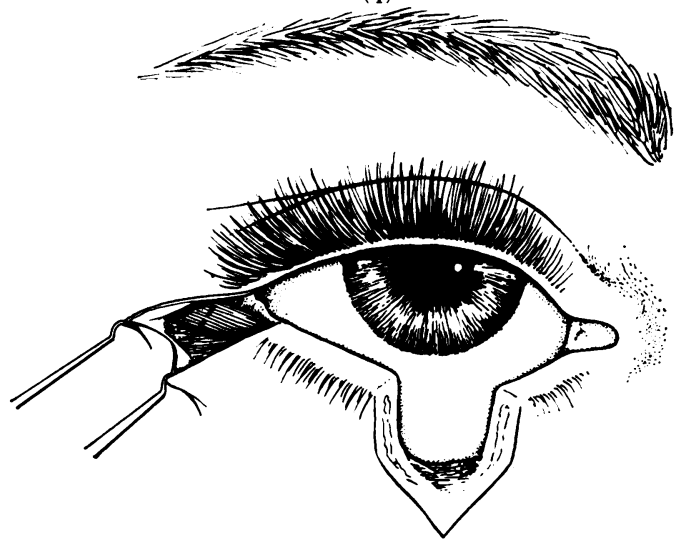

(6)

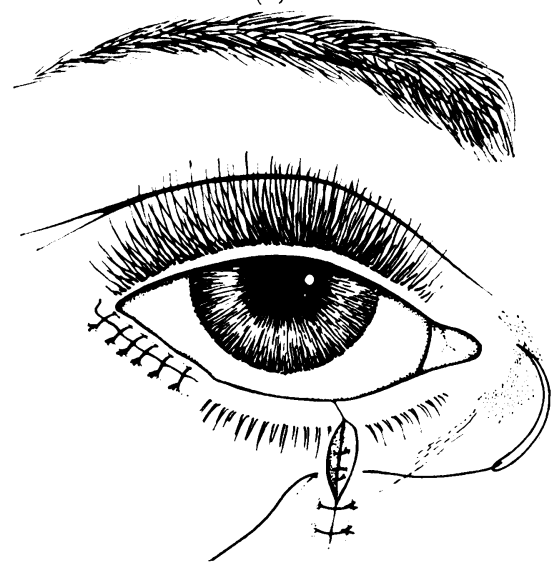

In the presence of larger defects (Fig.7), several techniques are available; lower eyelid reconstruction is best performed with a tarso-conjunctival slide from the upper lid (Fig. 8), the basic principles of which were elaborated by Landolt and Hughes (Hughes, 1954).

(7)
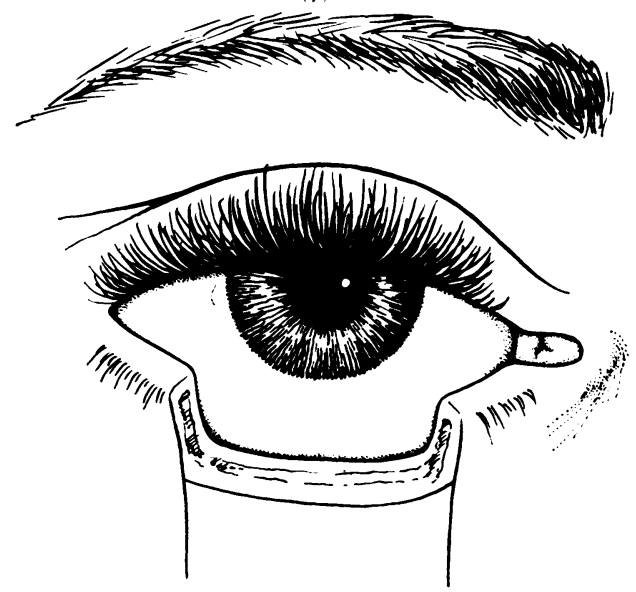

(8)

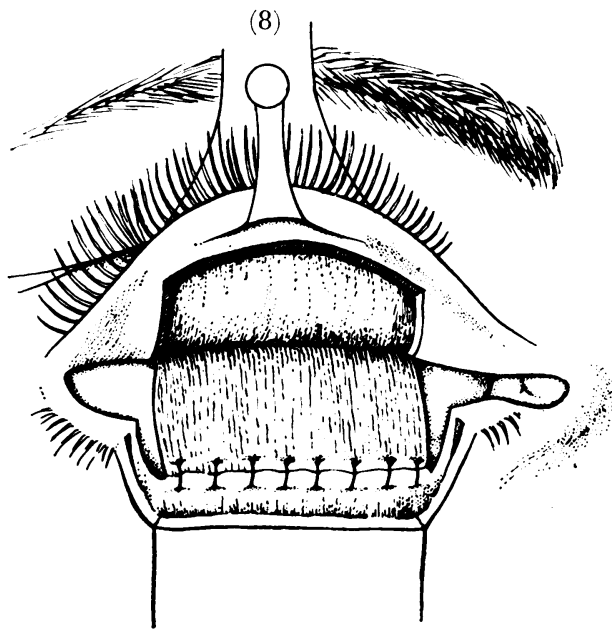


An Erhardt clamp inverts the upper lid and a flap of tarsus is prepared of horizontal span slightly less than the defect. Because it is imperative to preserve the integrity of the lid margin and the marginal arterial arcade, the initial incision is located $3 \mathrm{~mm}$. behind the border of the upper lid. Earlier writers described a splitting of this border, but such a modification can result in entropion and trichiasis with all their distressing sequelae, and is nowadays not recommended. Preparation of a flap which is too wide results in a sagging lower lid. Dissection of the tarsal flap is in the plane of the pretarsal fascia and is $\overparen{\otimes}$ carried upwards between Mueller's muscle and the levator palpebrae superioris aponeurosis. This results in a freely mobile flap which is taken down and sutured to the conjunctiva $\vec{\circ}$ of the lower fornix with interrupted 6-o chromic catgut sutures. Insufficient dissection: producing limitation of mobility results in vertical shortening and increased arching of the $\vec{\omega}$ upper eyelid postoperatively. Firmer anchorage is obtained by preparing a slot (Fig. 8) $\frac{O}{O}$. which allows the vertical margins of the advanced flap to interdigitate into the lower lid. Double-armed 6-o silk sutures tied anteriorly over cotton-wool pledgets accurately position the advanced tarsus in these grooves.

A full-thickness stamp skin graft is obtained from the retroauricular area or the upper lid of the other eye; this is fashioned and sutured in position with fine silk sutures and a telfa dressing is applied. Undermining an advancement flap with relaxation triangles is $\subseteq$ an adequate substitute (Fig. 9) where redundant skin is present in older patients.

(9)

(10)

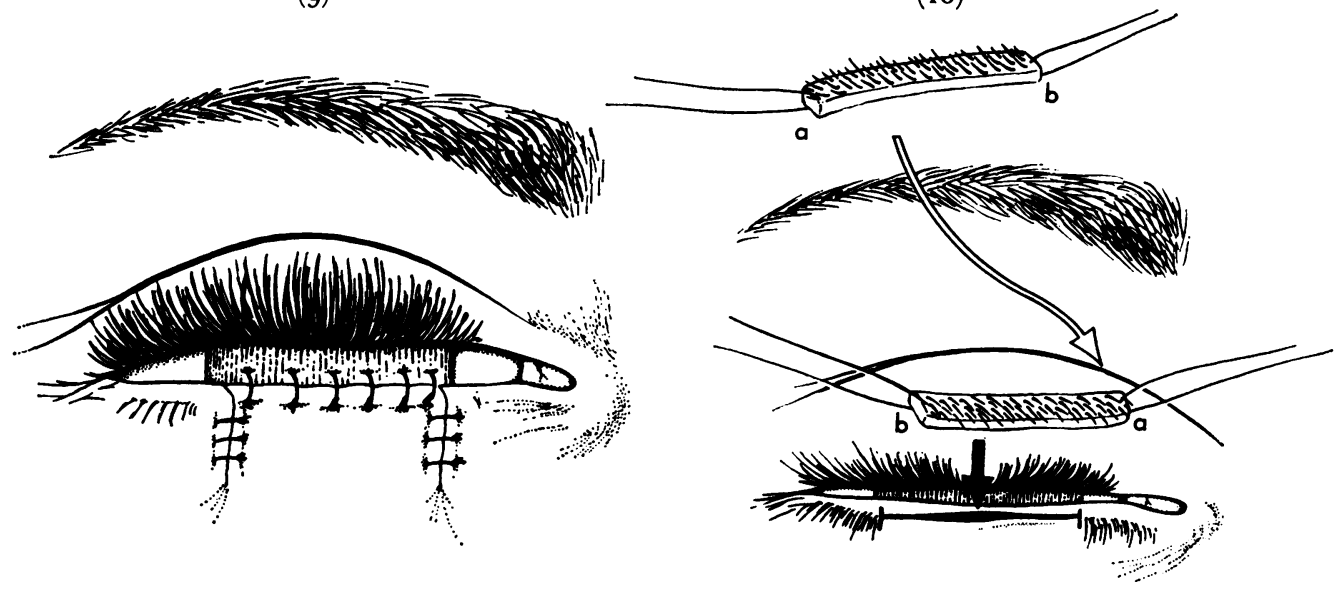

After this stage, a cilia graft can be undertaken at 4 weeks. This improves the appear- $\widetilde{N}$ ance and stability of the eyelid margin. An incision shaped like a capital $I$ is performed $N$ parallel to the new margin and the bed prepared (Fig. Io). The eyebrow is grasped $\mathfrak{\omega}^{\circ}$ firmly to produce haemostasis and a strip of full-thickness skin four lash roots in breadth is excised and sutured into its recipient site. After 3 weeks, the eyelids are separated by scissors section and any irregularities are trimmed into proper perspective. If the patient $\stackrel{\Phi}{\oplus}$ is not desirous of having cilia grafted, the blepharrhaphy may be opened 4 weeks postoperatively.

Large defects in the upper lid require a different approach, as the anatomical limitation of the tarsus of the lower lid makes it less readily available for advancement. A CutlerBeard bridge flap technique is then to be preferred (Cutler and Beard, 1955). Once 
again, preserving the integrity of the lid margin, a full-thickness incision is prepared as illustrated (Fig. I I). To control the line of incision, it is advisable to do this as a two-step procedure first from the external and then the internal aspect. A lid plate can be used to maintain stretch on the eyelid or a contact lens shell can act to protect the cornea. A satisfactory flap is prepared by adding the vertical parallel incisions, and this is taken under the bridge of the marginal strip (Fig. 12) to replace the upper lid defect. Interrupted $5^{-0}$ plain catgut sutures approximate the conjunctiva of the lower lid with conjunctiva and Mueller's muscle fibres above. The advanced orbicularis muscle is approximated to the levator aponeurosis and septum orbitale, and the skin layer is closed with silk sutures.

(II)

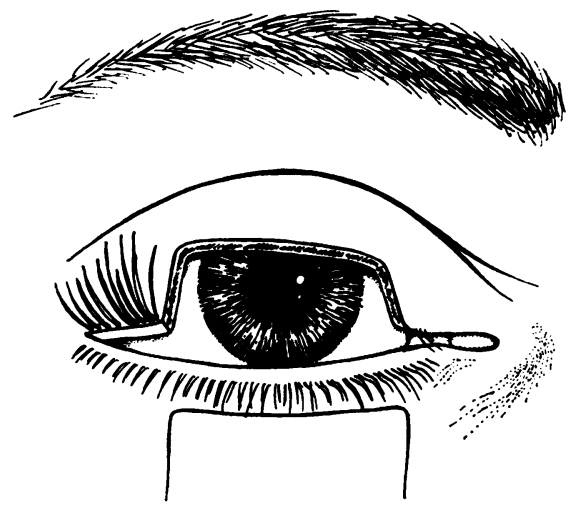

(12)

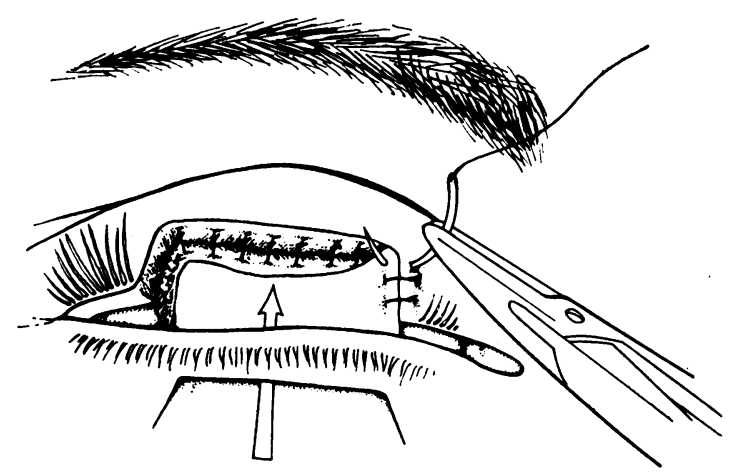

There is no need to close the inferior margin of the bridge flap as this will be covered rapidly by epithelial proliferation. Attempts to suture this are dangerous and impairment of circulation may result. The wound is dressed with telfa and a light pressure pad is applied. Too firm a dressing can produce circulatory embarrassment. The passage of time results in an increase in length of the advancement flap so that it is approximately doubled within 4 weeks. At this stage, grafting of cilia may be performed or, if this is not desired, the blepharrhaphy may be opened with scissors (Fig. 13) and the two components of the lower lid aligned and sutured in place.

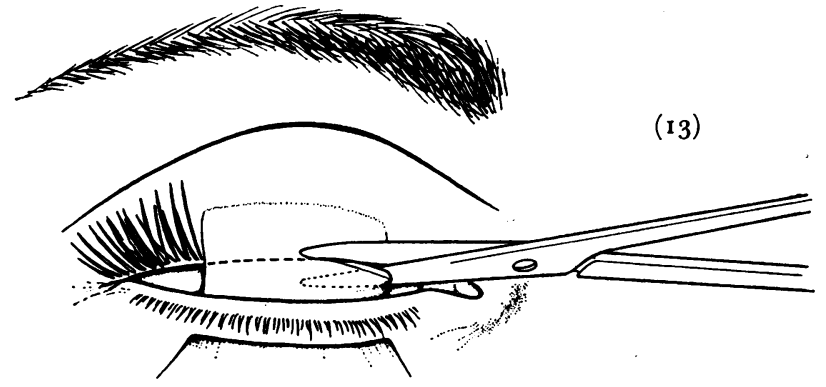

Excision of neoplasms involving the medial or lateral canthi pose special problems to the ophthalmologist, but basically the same principles are applied. In lateral canthal surgery (Fig. I4) a trick manoeuvre of fashioning a flap of orbital periosteum is recommended. This results in an artificial lateral canthal ligament which will give proper 
alignment of the palpebral fissure. Similarly, the stump of the medial canthal ligament can be used in surgery in this region (Fig. I5). Advancement skin flaps are also employed in these circumstances.

(14)

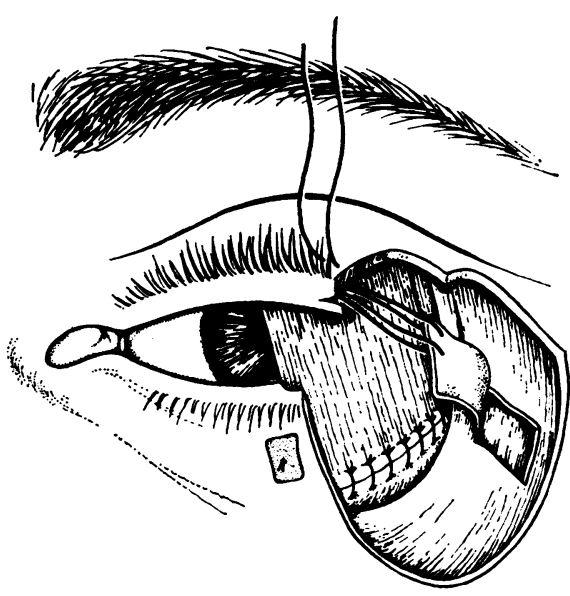

(15)

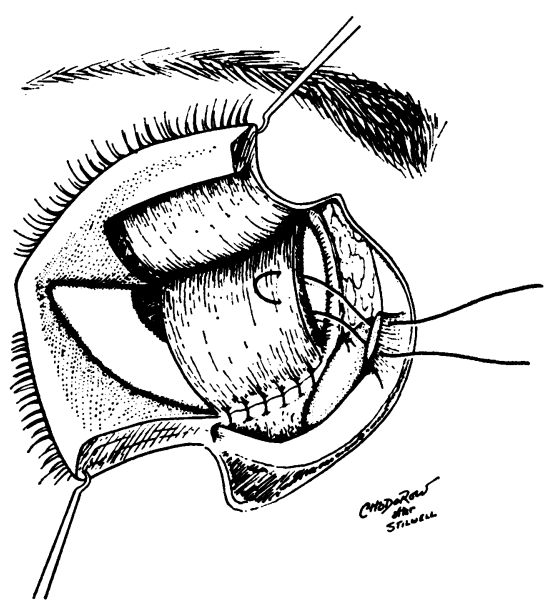

A number of ingenious pedicle grafts and rotation flap techniques are available for $\vec{\emptyset}$ reconstructive surgery of the eyelids (Mustardé, 1966). These can be utilized when it is preferable to keep the eye open as, for example, if the contralateral eye is diseased. The consensus of opinion of most ophthalmic plastic surgery clinics in North America, however, favours the earlier-mentioned techniques. These procedures pose no great technical problem to the ophthalmic surgeon with his intimate knowledge of the relevant regional $\stackrel{\circ}{\circ}$ anatomy. They are simple and brief techniques and have the advantage that the cosmetic $\overrightarrow{\vec{A}}$ appearance and texture are approximated, when the principle of replacement of eyelid by eyelid structure is followed. Scarring of tissue is less likely with skin of the orbital region as compared with adjoining zones. Large sliding or rotation flaps enhance this scarring potential in eyelid surgery, and they are especially not favoured for patients prone to keloid formation or for the Negro population. Advancement flaps offer assistance in partialthickness injuries to the eyelid and can be used effectively in suitable cases.

Large eyelid defects can also be closed by composite lid grafts (Fig. I6) obtained from the opposite upper lid. A full-thickness wedge of the eyelid is taken, inserted in the resected area, and sutured into place with interrupted fine silk sutures placed in the
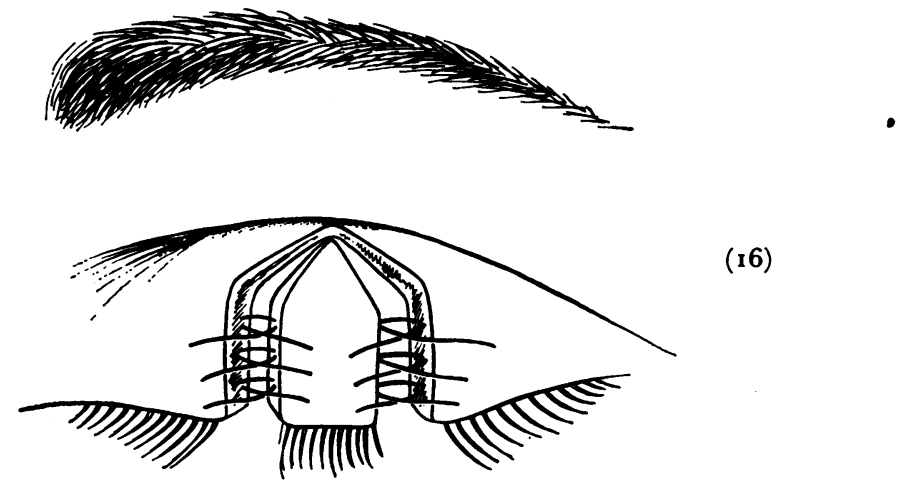
pretarsal fascia. Generally, the donor wedge of tissue is smaller in size than the recipient area. The skin incision is finally closed with 6-o silk sutures. This technique has recently become very popular, but is in fact a long-established one (Smith, I959).

The eminent John Wheeler (1939) often taught that ophthalmic plastic surgeons thought complexly and operated similarly. The fundamentals of the approach to reconstructive surgery of the eyelids presented above include a number of techniques available to the surgeon. Regardless of the one of his preference, the overriding principle should be that of simplicity in meeting the challenge of this increasing problem.

We are indebted to Dr. Alston P. Callahan of Birmingham, Alabama, for permission to reproduce photographs of his most interesting case.

\section{References}

CUtLer, N. L., and BeARd, c. (1955) Amer. F. Ophthal., 39, I

HUGHes, w. L. (1954) "Reconstructive Surgery of the Eyelids", 2nd ed., p. 185. Mosby, St. Louis MUSTARDÉ, J. c. (1966) "Repair and Reconstruction of the Orbital Region", p. i 16. Livingstone,

Edinburgh

Smitr, B. (1959) "Eyelid Surgery", Surg. Clin. N. Amer., 39, Symposium on Reconstructive Plastic Surgery, p. 367

wheeler, J. M. (1939) “Collected Papers of John Martin Wheeler, M.D., on Ophthalmic Subjects".

Columbia-Presbyterian-Medical Center, New York 